\title{
KLOOSTERMAN SUMS FOR CHEVALLEY GROUPS
}

\author{
ROMUALD DABROWSKI
}

\begin{abstract}
A generalization of Kloosterman sums to a simply connected Chevalley group $G$ is discussed. These sums are parameterized by pairs $(w, t)$ where $w$ is an element of the Weyl group of $G$ and $t$ is an element of a Q-split torus in $G$. The $S L(2, \mathbf{Q})$-Kloosterman sums coincide with the classical Kloosterman sums and $S L(r, \mathbf{Q})$-Kloosterman sums, $r \geq 3$, coincide with the sums introduced in [B-F-G, F, S]. Algebraic properties of the sums are proved by root system methods. In particular an explicit decomposition of a general Kloosterman sum over $\mathbf{Q}$ into the product of local $p$-adic factors is obtained. Using this factorization one can show that the Kloosterman sums corresponding to a toral element, which acts trivially on the highest weight space of a fundamental irreducible representation, splits into a product of Kloosterman sums for Chevalley groups of lower rank.
\end{abstract}

\section{INTRODUCTION}

The classical Kloosterman sums [Kl] are defined for any triplet of integers $m, n$, and $c, c>0$, by the formula

$$
S(m, n, c)=\sum_{\substack{x, y \bmod c \\ x y=1(\bmod c)}} e^{2 \pi i} \frac{m x+n y}{c} .
$$

These sums are connected with many problems in number theory (for an overview of number theoretical applications of Kloosterman sums see [D-I]. In [BF-G, F, S] generalizations of Kloosterman sums to certain trigonometric sums related to the Bruhat decomposition of $G L(n)$ are introduced. These sums are defined as follows. Let $T$ and $U_{+}$denote the diagonal and the upper triangular unipotent subgroups of $G L(n, \mathbf{R})$, respectively, and let $\theta_{1}, \theta_{2}$ be characters of $U_{+}$that are trivial on the subgroup $U_{+}(\mathbf{Z})$ consisting of elements of $U_{+}$with integral matrix coefficients. Let $t \in T$ and let $\omega$ be a generalized permutation matrix in $G L(n, \mathbf{R})$ (with \pm 1 nonzero entries) corresponding to a Weyl group element $w$. Then the Kloosterman sum corresponding to the data $\theta_{1}, \theta_{2}, t$ and $w$ is defined by the formula

$$
S\left(\theta_{1}, \theta_{2} ; t, w\right)=\sum \theta_{1}\left(b_{1}\right) \theta_{2}\left(b_{2}\right)
$$

where the summation ranges over representatives $b_{1} t \omega b_{2}$ of distinct elements in finite set $U_{+}(\mathbf{Z}) \backslash U_{+}(\mathbf{Q}) t \omega U_{+}(\mathbf{Q}) \cap S L(n+1, \mathbf{Z}) / U_{w}(\mathbf{Z})$ where $U_{w}(\mathbf{Z})=$

Received by the editors December 13, 1989 and, in revised form, February 25, 1991.

1980 Mathematics Subject Classification (1985 Revision). Primary 10G05, 20G05; Secondary $14 \mathrm{~L} 17$. 
$U(\mathbf{Q}) \cap \omega^{-1} U(\mathbf{Z}) \omega$. This sum is well defined if certain conditions are imposed on $\theta_{1}, \theta_{2}$ and $t$. In particular $t$ has to be of the form

$$
t=\operatorname{diag}\left(1 / c_{1}, c_{1} / c_{2}, c_{2} / c_{3}, \ldots, c_{n-2} / c_{n-1}\right),
$$

where $c_{1}, c_{2}, \ldots, c_{n-1}$ are nonzero integers. One easily shows that $G L(2)$ sums coincide with the classical Kloosterman sums. The related Kloosterman zeta function is defined by the formula

$$
Z_{w}\left(\theta_{1}, \theta_{2} ; s\right)=\sum_{t} S\left(\theta_{1}, \theta_{2} ; t, w\right) t^{-s} .
$$

The summation ranges over all $t=\operatorname{diag}\left(1 / c_{1}, c_{1} / c_{2}, c_{2} / c_{3}, \ldots, c_{n-2} / c_{n-1}\right)$, where $c_{1}, c_{2}, \ldots, c_{n-1}$ are positive integers, $s=\left(s_{1}, s_{2}, \ldots, s_{n-1}\right)$ is an $(n-1)$-tuple of complex numbers and $t^{-s}=c_{1}^{-n s_{1}} c_{2}^{-n s_{2}} \cdots c_{n-1}^{-n s_{n-1}}$. It is observed in [B-F-G] that the generalized Ramanujan conjecture about Fourier coefficients of nonholomorphic cusp forms follows if the zeta functions $Z_{w}\left(\theta_{1}, \theta_{2} ; s\right)$ have a suitable meromorphic continuation.

In hope of obtaining better understanding of Kloosterman sums (1.1) we develop a theory of Kloosterman sums for a Chevalley group scheme $G$. These sums are associated with the following data: a field $k$ and a subring $R$ of $k$, a subset $C$ of positive roots in the root system of $G$, an element $t$ of the split torus in the group $G(k)$ of $k$-points of $G$ and a pair of characters $\phi$ and $\psi$ of certain unipotent subgroups $U_{-}(k)$ and $U_{C}(k)$ of $G(k) \quad(\phi$ and $\psi$ are required to be trivial on subgroups $U_{-}(R)$ and $U_{C}(R)$, respectively). The Kloosterman sum corresponding to the data is

$$
S_{C}(t, \phi, \psi)=\sum \phi\left(u^{\prime}\right) \psi(u)
$$

where the summation ranges over a full set of representatives of

$$
U_{-}(R) \backslash U_{-}(k) t U_{C}(k) \cap G(R) / U_{C}(R)
$$

of the form $u^{\prime} t u$, where $u^{\prime} \in U_{-}(k)$ and $u \in U_{C}(k)$ (we show in what circumstances the sum is finite). It turns out that the sums $S_{C}(t, \phi, \psi)$ are generalizations the sums defined by (1.2). We prove various algebraic properties of sums (1.2) by root system methods. In particular, we obtain a decomposition of a generalized Kloosterman sum over $\mathbf{Q}$ into a product of "local" Kloosterman sums defined over $\mathbf{Q}_{p}$. Using this decomposition we give an explicit decomposition of Kloosterman sums into a product of classical Kloosterman sums in some special cases.

My thanks are due to D. Goldfeld for an introduction to the subject of Kloosterman sums and encouragement during my work on the paper. I am also thankful to V. Deodhar, B. Haboush and H. Jacquet for useful discussions.

\section{Generalized Kloosterman Sets and sums}

Let $L$ be a semisimple Lie algebra over $C$ of rank $r$. Suppose that $H$ is a fixed Cartan subalgebra of $L, \Phi \subset H^{*}$ is the root system corresponding to $H$ with the Weyl group $W, \Delta$ is a fixed basis of $\Phi, \Phi^{+}=\left\{\alpha_{1}, \alpha_{2}, \ldots, \alpha_{m}\right\}$ is the set of positive roots, $h_{i}, 1 \leq i \leq r, x_{\alpha}, \alpha \in \Phi$, is a fixed Chevalley basis for $L$. We also let $\Lambda$ denote the lattice of integral weights of $\Phi$ (for definitions see $[\mathrm{H}]$ ). Let $G$ denote the ("simply connected") Chevalley group scheme $[\mathrm{Ch}, \mathrm{K}, \mathrm{Bo}]$ associated with the above data. Let $k$ be a field. Then 
the group $G(k)$ of $k$-points of $G$ can be described as follows. Let $V$ be any complex, faithful representation of $L$, such that $Z$-linear span of the set of weights of $V$ coincides with $\Lambda$. Recall that $\mu \in H^{*}$ is a weight of $V$ if

$$
V_{\mu}=\{v \in V, h v=\mu(h) v\} \neq\{0\} .
$$

Let $M$ be any admissible lattice in $V[\mathrm{H}, \S 27]$ and let $V(k)=k \otimes_{Z} M$. For any $\alpha \in \Phi$ and $\xi \in k$ let $U_{\alpha}(\xi)$ be an element of $\operatorname{End}_{k}(V(k))$ given by the formula

$$
U_{\alpha}(\xi)(1 \otimes v)=\sum_{m=1}^{\infty} \xi^{m} \otimes \frac{x_{\alpha}^{m}}{m !} v
$$

where $v$ is any element of $M$. Then $U_{\alpha}(\xi)$ is in fact an automorphism of $V(k)$. Moreover

$$
\begin{aligned}
& U_{\alpha}(\xi)^{-1}=U_{\alpha}(-\xi) \text { and } U_{\alpha}(\xi+\eta)=U_{\alpha}(\xi) U_{\alpha}(\eta) \\
& U_{\alpha}(\xi) U_{\beta}(\eta) U_{\alpha}(-\xi) U_{\beta}(-\eta)=\prod U_{i \alpha+j \beta}\left(c_{i, j} \xi^{i} \eta^{j}\right)
\end{aligned}
$$

for any $\alpha, \beta \in \Phi, \alpha+\beta \neq 0, \xi, \eta \in k$. The product on the right-hand side of (2.1) is taken over all roots of the form $i \alpha+j \beta \quad(i, j$ are positive integers) arranged in some fixed ordering, $c_{i, j}$ are integers depending on $\alpha, \beta$, and the chosen ordering, but not on $\eta$ and $\xi$. Moreover, $\left[x_{\alpha}, x_{\beta}\right]=c_{11} x_{\alpha+\beta}$ if $\alpha+\beta$ is a root (for proofs see [St]). Then $G(k)$ coincides with the subgroup of $\operatorname{Aut}_{k}(V(k))$, generated by the set $\left\{U_{\alpha}(\xi) ; \alpha \in \Phi, \xi \in k\right\}$. We also let $T(k)$ denote the split torus in $G(k)$.

Let $V^{\prime}$ be any complex finite dimensional representation of $L$, and let $M^{\prime}$ be an admissible lattice in $V^{\prime}$. Then $V^{\prime}(k)=k \otimes_{Z} M^{\prime}$ admits a $k$-linear action of $G(k)$ such that

$$
U_{\alpha}(\xi)(1 \otimes v)=\sum_{m=1}^{\infty} \xi^{m} \otimes \frac{x_{\alpha}^{m}}{m !} v
$$

for any $\alpha \in \Phi$ and $\xi \in k$ and $v \in M^{\prime}$. For any weight $\mu$, the corresponding $T(k)$-weight space is $V_{\mu}^{\prime}(k)=k \otimes_{Z}\left(V_{\mu}^{\prime} \cap M^{\prime}\right)$. We recall how $T(k)$ and $U_{\alpha}(\xi)$ act on spaces $V_{\mu}^{\prime}(k), \mu \in \Lambda$. Suppose that $v \in V_{\mu}^{\prime}(k)$. Then

$$
t v=t(\mu) v \text { for any } t \in T(k),
$$

where the map $(\mu, t) \rightarrow t(\mu)$ defines a group homomorphism form $\Lambda \times T(k)$ to $k^{*}$ (the homomorphism is independent of $V^{\prime}$ and $M^{\prime}$ ). If $\alpha \in \Phi, \xi \in k$, then

$$
U_{\alpha}(\xi) v-v \in \bigoplus_{n \geq 1} V_{\mu+n \alpha}^{\prime}(k) .
$$

Let $R$ be any subring of $k$ containing 1 . Then $G(R)=\left\{g \in G(k) ; g\left(R \otimes_{Z} M\right)=R \otimes_{Z} M\right.$, for any admissible lattice $M$ in any complex representation of $L\}$.

A result of Chevalley [Ch] states that

$$
G(R)=\left\{g \in G(k) ; g\left(R \otimes_{Z} M\right)=R \otimes_{Z} M\right\}
$$

where $M$ is an admissible lattice in a faithful representation $V$ of $L$ such that the Z-linear span of the weights of $V$ is $\Lambda$. 
Let $C$ be subset of $\Phi$. $C$ is said to be closed if $\alpha, \beta \in C, \alpha+\beta \in \Phi$, implies $\alpha+\beta \in C$. A subset $I$ of a closed set $C$ is called an ideal of $C$ if $\alpha \in I, \beta \in C, \alpha+\beta$ implies $\alpha+\beta \in I$.

Example. Let $w$ be an element in the Weyl group $W$. Then

$$
P(w)=\left\{\alpha \in \Phi^{+} ; w(\alpha) \in \Phi^{-}\right\}
$$

is a closed subset of $\Phi^{+}$.

Let $C$ be a closed set and let $U_{C}(R)$ denote the subgroup of $G(k)$ generated by elements $U_{\alpha}(\xi), \alpha \in C, \xi \in R$ (we write $U_{+}=U_{\Phi_{+}}$and $U_{-}=U_{\Phi_{-}}$). Clearly, $U_{C}(R) \subseteq G(R)$. If $C \cap(-C)=\varnothing,(2.1)$ implies that any element $u$ of $U_{C}(R)$ can be written uniquely as the product

$$
u=U_{\alpha_{1}^{\prime}}\left(\xi_{1}\right) U_{\alpha_{2}^{\prime}}(\xi) \cdots U_{\alpha_{d}^{\prime}}\left(\xi_{d}\right)
$$

where $\left(\alpha_{1}^{\prime}, \alpha_{2}^{\prime}, \ldots, \alpha_{d}^{\prime}\right), d=\#(C)$, is any ordering of $C$ and $\xi_{1}, \xi_{2}, \ldots, \xi_{d} \in$ $R\left(\xi_{1}, \xi_{2}, \ldots, \xi_{d}\right.$ are called the coordinates of $\left.u\right)$.

Definition. For any $t \in T(k)$ we define the sets

$$
\begin{gathered}
Y_{C}(t)=\left\{g \in G(R) ; g=x t y, x \in U_{-}(k), y \in U_{C}(k)\right\}, \\
Y(t)=Y_{\Phi^{+}}(t)=U_{-}(k) t U_{+}(k) \text { and } K_{C}(t)=U_{-}(R) \backslash Y_{C}(t) / U_{C}(R) .
\end{gathered}
$$

If $w$ is an element of the Weyl group, we write $Y_{w}=Y_{P(w)}$ and $K_{w}=K_{P(w)}$. $K_{w}(t)$ is called a Kloosterman set. The following proposition summarizes basic properties of sets $Y_{C}$ and $K_{C}$.

Proposition 2.2. (i) If $C \subset C^{\prime}$ then $Y_{C}(t) \subset Y_{C^{\prime}}(t)$ and $K_{C}(t) \subset K_{C^{\prime}}(t)$ (under the obvious inclusion maps).

(ii) $t \neq t^{\prime}$ then $Y_{C}(t) \cap Y_{C^{\prime}}\left(t^{\prime}\right)=\varnothing$ for any $C, C^{\prime}$. If $t^{-1} t^{\prime} \in T(R)=T(k) \cap$ $G(R)$ then the elements of $K_{C}(t)$ and $K_{C}\left(t^{\prime}\right)$ are in a one-to-one correspondence via the map $y \rightarrow y t^{-1} t^{\prime}$.

(iii) $Y_{C}(t) \neq \varnothing$ then $t(\lambda) \in R$ for all dominant weights $\lambda \in \Lambda$.

(iv) Let $u^{\prime} t u \in Y(t)$ for some $t \in k$, and assume $u^{\prime}=\prod_{\alpha \in \Phi^{+}} U_{-\alpha}\left(\xi_{-\alpha}\right)$ $u=\prod_{\alpha \in \Phi^{+}} U_{\alpha}\left(\xi_{\alpha}\right)$ (products taken in a fixed order), $\xi_{-\alpha}, \xi_{\alpha}$ in $k$. Let $\rho=$ $\left(\sum_{\alpha \in \Phi^{+}} \alpha\right) / 2$ and let $\left\{\lambda_{\alpha} ; \alpha \in \Delta\right\}$ be the set of fundamental weights relative to $\Delta$. Then there exists a positive integer $N$ such that $t(N \rho) \xi_{-\alpha}$ and $t(N \rho) \xi_{\alpha}$ are in $R$ for all $\alpha \in \Phi^{+}$. Moreover $t\left(\lambda_{\alpha}\right) \xi_{-\alpha}$ and $t\left(\lambda_{\alpha}\right) \xi_{\alpha}$ are in $R$ for all $\alpha \in \Delta$.

(v) Let $v$ denote the quotient (abelian group) homomorphism $k \rightarrow k / R$. Assume that $R$ satisfies

$$
\text { For any } x \in R \text { the set } v(\{y \in k ; x y \in R\}) \text { is finite. }
$$

Then $K_{C}(t)$ is either finite or empty.

Proof. (i) and (ii) are straightforward. For (iii) let $\lambda$ be a dominant weight and let $V$ be the irreducible representation of weight $\lambda$ with a highest weight vector $v$. We can assume that $v$ is contained in a $\mathbf{Z}$-basis of an admissible lattice $M$ of $V[H, \S 27]$. Therefore $1 \otimes v$ belongs to a free $R$-basis of $R \otimes Z M$. For any $g \in G(k)$, we let $f(g)$ denote the $1 \otimes v$-component of $g(1 \otimes v)$. If $g=u^{\prime} t u \in Y(t)$ then $f(g)=t(\lambda) \in R$ by properties of the action of $T(k)$ and $U_{ \pm}(k)$ on weight spaces of $V(k)$.

Part (iv). We need the following fact due to Chevalley [Ch, Bo]. 
Lemma 2.3. For any $\alpha \in \Phi^{+}$, there exist functions $F_{ \pm \alpha}$ defined on $G(k)$ with values in $k$ and polynomials $P_{ \pm \alpha}$ with integer coefficients in respective indeterminants $x_{ \pm \beta}, 0<\beta<\alpha$, such that

(i) $P_{ \pm \alpha}=0$ if $\alpha$ is a simple root,

(ii) if $g \in G(R)$ then $F_{ \pm \alpha}(g) \in R$,

(iii) if $g=U_{-\alpha_{1}}\left(\eta_{-\alpha_{1}}\right) U_{-\alpha_{2}}\left(\eta_{-\alpha_{2}}\right) \cdots U_{-\alpha_{m}}\left(\eta_{-\alpha_{m}}\right) t U_{\alpha_{1}}\left(\eta_{\alpha_{1}}\right) U_{\alpha_{2}}\left(\eta_{-\alpha_{2}}\right) \cdots$ $U_{-\alpha_{m}}\left(\eta_{\alpha_{m}}\right)$ where $t \in T(k)$ and $\eta_{\alpha} \in k, \alpha \in \Phi$, then

$$
F_{ \pm \alpha}(g)=t(2 \rho) \eta_{ \pm \alpha}+P_{ \pm \alpha}\left(\eta_{ \pm \beta}, 0<\beta<\alpha\right) .
$$

The lemma implies the first part of (iv) by induction on the height of a given root (height of a root is the sum of its simple root components). For the second part we consider a simple root $\alpha$ and the irreducible representation $V$ of the highest weight $\lambda_{\alpha}$. Let $v$ be the highest weight vector. Let $v_{\alpha}$ be a nonzero vector in the weight space $V_{\sigma_{\alpha}\left(\lambda_{\alpha}\right)}=V_{\lambda_{\alpha}-\alpha}$, where $\sigma_{\alpha} \in W$ is the reflection corresponding to $\alpha$. We may assume that $v$ and $v_{\alpha}$, belong to a Z-basis of an admissible lattice $M$ in $V$ (see [H, 27.1]). Therefore $1 \otimes v$ and $1 \otimes v_{\alpha}$ belong to a basis of $k \otimes_{Z} M$. For any $g \in G(k)$, we let $f_{\alpha}(g)$ denote the $1 \otimes v_{\alpha^{-}}$ component of $g(1 \otimes v)$. A calculation based on properties of weight spaces [H, 20.1] shows $f_{\alpha}\left(u^{\prime} t u\right)=t\left(\lambda_{\alpha}\right) \xi_{-\alpha} \in R$. Finally, let $\vartheta$ denote the automorphism of $G(k)$ induced by the automorphism of $\Phi$ sending a root $\alpha$ to $-\alpha$. Then $f_{\alpha}\left(\vartheta\left(u^{\prime} t u\right)^{-1}\right)=f_{\alpha}\left(\vartheta\left(u^{-1}\right) t \vartheta\left(\left(u^{\prime}\right)^{-1}\right)\right)=-t\left(\lambda_{\alpha}\right) \eta_{\alpha} \in R$.

Part (v). In view of (iv) it is enough to prove the following lemma.

Lemma 2.4. Let $\left(\alpha_{1}, \alpha_{2}, \ldots, \alpha_{m}\right)$ be an ordering of $\Phi^{+}$such that $\left\{\alpha_{1}, \alpha_{2}, \ldots\right.$, $\left.\alpha_{q}\right\}, 1 \leq q \leq m$, is an ideal in $\Phi^{+}$. For any finite subset $S$ of $k / R$ we set

$$
Y_{S}^{q}=\left\{\prod_{i=1}^{q} U_{\alpha_{i}}\left(\xi_{i}\right) ; v\left(\xi_{i}\right) \in S\right\} .
$$

If $R$ satisfies property $(\mathrm{P} 1)$ then $\pi\left(Y_{S}^{q}\right)$ is finite (here $\pi: U_{+}(k) \rightarrow U_{+}(k) / U_{+}(R)$ is the quotient homomorphism).

Proof of the lemma. The lemma is trivial in the case $q=1$. Assume $q>1$. For any $\xi \in k$ we define $Z_{S}^{q}(\xi)=Y_{S}^{q-1} U_{\alpha_{q}}(\xi)$. The assumptions of the lemma imply that $\pi\left(Y_{S}^{q}\right) \subset \bigcup_{j=1}^{d} \pi\left(Z_{S}^{q}\left(\xi_{j}\right)\right)$ for some $\xi_{j} \in k$. Consequently it is enough to show that $\pi\left(Z_{S}^{q}(\xi)\right)$ is finite for any $\xi \in k$. We observe that $U_{\alpha_{q}}(-\xi) Z_{S}^{q}(\xi) \subset$ $Y_{S^{\prime}}^{q-1}$ for some finite set $S^{\prime}$ of $k / R$ since by identity (2.1) the coordinates of elements in $U_{\alpha_{q}}(-\xi) Z_{S}^{q}(\xi)$ are polynomial functions of the coordinates of elements in $Y_{S}^{q}$, and the polynomials involved depend only on the ordering of the roots. By induction on $q$ we obtain $\#\left(\pi\left(Z_{S}^{q}(\xi)\right)\right)=\#\left(\pi\left(U_{\alpha_{q}}(-\xi) Z_{S}^{q}(\xi)\right)\right) \leq$ $\#\left(\pi\left(Y_{S^{\prime}}^{q-1}\right)\right)<\infty$.

The next proposition will allow us to prove properties of Kloosterman sets by induction on the rank of the Lie algebra. We recall that $\Phi$ is a subset of a real vector space $E$ equipped with a scalar product $($,$) . As usual we write$ $\langle\lambda, \mu\rangle=\frac{2(\lambda, \mu)}{(\mu, \mu)}$ for any elements $\lambda, \mu \in E$, such that $\mu \neq 0$. With this notation

$$
\Lambda=\{\lambda \in \Lambda ;\langle\lambda, \alpha\rangle \in \mathbf{Z} \text { for all } \alpha \in \Phi\}
$$

and the fundamental weights $\lambda_{\alpha}, \alpha \in \Delta$, are defined by conditions

$$
\left\langle\lambda_{\alpha}, \gamma\right\rangle=\delta_{\alpha, \gamma} \quad \text { for all } \alpha, \gamma \in \Delta \text {. }
$$


Proposition 2.5. Suppose that $R \subset k$ satisfies the property

$\left(\mathrm{P}_{2}\right)$ Let $x \in k$. If $x^{n} \in R$ for some nonnegative integer $n$ then $x \in R$. Let $u^{\prime} t u \in Y_{C}(t)$ and let $t\left(\lambda_{\alpha}\right)$ be a unit in $R$ for some $\alpha \in \Delta$. Let $U_{C^{\prime}}(k)$ (resp. $U_{-}^{\prime}(k)$ ) denote the subgroup of $U_{+}(k)$ (resp. $U_{-}^{\prime}(k)$ ) generated by elements $U_{\beta}(\eta), \eta \in k, \beta \in C$ (resp. $\left.U_{\beta}(\eta), \eta \in k, \beta \in \Phi^{-}\right)$such that $\left\langle\lambda_{\alpha}, \beta\right\rangle=0$. Then there exist elements $y \in U_{-}(R)$ and $x \in U_{C}(R)$ such that $y u^{\prime} \in U_{-}^{\prime}(k)$ and $u x \in U_{C^{\prime}}(k)$.

Proof. We need the following general fact about root systems.

Lemma 2.6 (Special filtration of sets of roots). For a simple root $\alpha$ we let

$$
B=B(\alpha)=\left\{\beta \in \Phi^{+} ;\left\langle\lambda_{\alpha}, \beta\right\rangle>0\right\}
$$

( $B$ is just the set of all positive roots with nonzero $\alpha$-component). We define $B_{1}=\{\alpha\}$ and, inductively, we let $B_{n}$ denote the set of elements $\beta$ in $B$ such that if $\left\langle\lambda_{\alpha}, \beta\right\rangle \beta=\sum_{j} \beta_{j}, \beta_{j} \in B$, then either $\beta_{j}=\beta$ for all $j$ or $\beta_{j} \in B_{n-1}$ for some $j$ (observe that $B_{n-1} \subseteq B_{n}$ ). Then $B=\bigcup\left\{B_{n} ; n \geq 1\right\}$.

Sketch of a proof. Clearly, it is enough to consider irreducible root systems. In the case of a root system of type $G_{2}$, the lemma can be verified by inspection. In remaining cases, the lemma follows, since $\{\beta \in B ; \mathrm{ht}(\beta) \leq n\} \subseteq B_{n}$ for all $n, n=1,2, \ldots$ (here, $\operatorname{ht}(\beta)=\sum_{\alpha \in \Delta} c_{\alpha}$, if $\left.\beta=\sum_{\alpha \in \Delta} c_{\alpha} \alpha\right)$. The above statement can be derived from the following two facts:

(1) In any root system positive roots of equal height are linearly independent over the reals.

(2) If $\Phi \subseteq E$ is an irreducible root system of type different than $G_{2}$, than for any positive root $\beta$ there exists a real valued function $f$ defined in $E$, such that:

(i) $f\left(\gamma+\gamma^{\prime}\right) \leq f(\gamma)+f\left(\gamma^{\prime}\right)$ and $f(q \gamma)=q f(\gamma)$ for any $\gamma, \gamma^{\prime} \in E$ and $q=0,1,2, \ldots$

(ii) $f(\beta) \neq 0$ and $f(\beta) \geq f\left(\beta^{\prime}\right)$ for all roots $\beta^{\prime}$ with $\mathrm{ht}\left(\beta^{\prime}\right) \geq \mathrm{ht}(\beta)$.

We now come back to the proof of Proposition 2.5. For a fixed $\alpha \in \Delta$, we let

$$
B(\alpha)=\left\{\beta \in \Phi^{+} ;\left\langle\lambda_{\alpha}, \beta\right\rangle>0\right\}=\left\{\beta_{1}, \beta_{2}, \ldots, \beta_{N}\right\}
$$

where the ordering $\left(\beta_{1}, \beta_{2}, \ldots, \beta_{N}\right)$ of $B(\alpha)$ is consistent with the filtration $\left\{B_{n}\right\}$ of the lemma (i.e. if $\beta_{i} \in B_{n}$ and $\beta_{j} \notin B_{n}$ for some $n$ then $i<j$ ). Let $g=u^{\prime} t u \in Y(t)$ where $u^{\prime}=U_{-\beta_{1}}\left(\xi_{1}\right) U_{-\beta_{2}}\left(\xi_{2}\right) \cdots U_{-\beta_{N}}\left(\xi_{N}\right) u_{1}$ for some $u_{1} \in$ $U_{-}^{\prime}(k)$ and $u=u_{2} U_{\beta_{i_{M}}}\left(\eta_{M}\right) U_{\beta_{i_{M-1}}}\left(\eta_{M-1}\right) \cdots U_{\beta_{i_{1}}}\left(\eta_{1}\right) u_{2}$ where, $i_{1} \leq i_{2} \leq \cdots \leq$ $i_{M},\left\{\beta_{i_{1}}, \beta_{i_{2}}, \ldots, \beta_{i_{M}}\right\}=B(\alpha) \cap C$ and $u_{2} \in U_{C^{\prime}}(k)$. It is enough to show that $\xi_{i}, 1 \leq i \leq N$ and $\eta_{i}, 1 \leq i \leq M$, are in $R$. We will prove this using the following general fact about weight spaces of a fundamental representation of $L$.

Lemma 2.7. Let $\alpha \in \Delta, \beta \in \Phi^{+}, c=\left\langle\lambda_{\alpha}, \beta\right\rangle$ and let $V$ be the irreducible representation of weight $\lambda_{\alpha}$. Then $\frac{x_{\beta}^{c}}{c !} \frac{x_{-\beta}^{c}}{c !} v=v$ on $V_{\lambda_{\alpha}}$. Consequently $\frac{x_{-\beta}^{c}}{c !}$ maps $V_{\lambda_{\alpha}}$ isomorphically onto $V_{\lambda_{\alpha}-c \beta}$ since $\sigma_{\beta}\left(\lambda_{\alpha}\right)=\lambda_{\alpha}-c \beta$ and $\operatorname{dim}\left(V_{\lambda_{\alpha}}\right)=$ $\operatorname{dim}\left(V_{\lambda_{\alpha}-c \beta}\right)=1$. Moreover, if $\mu=\lambda_{\alpha}-\sum_{\beta \in \Delta-\{\alpha\}} n_{\beta} \beta$ where $n_{\beta}$ are nonnegative integers, not all equal to zero, then $V_{\mu}=\{0\}$. 
Proof of the lemma. The first assertion follows directly from the identity

$$
\frac{x_{\beta}^{c}}{c !} \frac{x_{-\beta}^{a}}{a !}=\sum_{k=0}^{\min (a, c)} \frac{x_{-\beta}^{(a-k)}}{(a-k) !}\left(\begin{array}{c}
h_{\beta}-a-c+2 k \\
k
\end{array}\right) \frac{x_{\beta}^{(c-k)}}{(c-k) !}
$$

where $\beta$ is any positive root and $h_{\beta}=\left[x_{\beta}, x_{-\beta}\right][\mathrm{H}, \S 26.2]$. The second assertion follows from the Freudenthal's multiplicity formula $[H, \S 26.2]$.

We continue the proof of Proposition 2.5. Let $v$ be a highest weight vector contained in a basis of an admissible lattice $M$ of $V$. Since $M=\bigoplus_{\mu \in \Lambda} M \cap V_{\mu}$ (see $[\mathrm{H}, \S 27]$ ) we can assume that the basis consists of weight vectors. For any $\beta_{i} \in B, i=1,2, \ldots, N$, let $v_{i}$ denote a basis vector of weight $\sigma_{\beta_{i}}\left(\lambda_{\alpha}\right)=$ $\lambda_{\alpha}-\left\langle\lambda_{\alpha}, \beta_{i}\right\rangle \beta_{i}$. For any $g \in G(k)$, let $f_{i}(g)$ denote the $1 \otimes v_{i}$ component of $g(1 \otimes v)$. By the definition of sets $B_{i}, i=1,2, \ldots, N$, one obtains $f_{1}(g)=$ $t\left(\lambda_{\alpha}\right) \xi_{1}$ and inductively

$$
\begin{aligned}
& f_{j}\left(U_{-\beta_{j-1}}\left(-\xi_{j-1}\right) U_{-\beta_{j-2}}\left(-\xi_{j-2}\right) \cdots U_{-\beta_{1}}\left(-\xi_{1}\right) g\right) \\
& \quad=t\left(\omega_{\alpha}\right) f_{j}\left(U_{-\beta_{j}}\left(\xi_{j}\right) U_{-\beta_{j+1}}\left(\xi_{j+1}\right) \cdots U_{-\beta_{N}}\left(\xi_{N}\right)\right)=t\left(\lambda_{\alpha}\right) \xi_{j}^{\left\langle\lambda_{\alpha}, \beta_{j}\right\rangle}
\end{aligned}
$$

for $j=1,2, \ldots, N$. Therefore property (P2) implies $\xi_{j} \in R$ for all $j$. Similarly, we obtain $\eta_{j} \in R$ for all $j$, replacing $g$ with $\vartheta\left(g^{-1}\right)$, where $\vartheta$ is the automorphism of $G(k)$ induced by the automorphism of the root system $\Phi$ sending a root $\beta$ to $-\beta$.

Let $J$ be a subset of the set of simple reflections and let $\Delta_{J} \subset \Delta$ be the corresponding set of simple roots. Let $\Phi_{J}$ denote the root system obtained by taking the set of roots in $\Phi$ which are linear combinations of roots in $\Delta_{J}$. Let $W_{J}$ denote the Weyl group of $\Phi_{J}$ (for a discussion of subgroups of the Weyl group, or more generally a Coxeter group, see [D1, D2]. Then the groups $G^{J}(k), U_{ \pm}^{J}(k)$, and $T^{J}(k)$, associated with $\Phi_{J}$, can be naturally identified with subgroups of $G(k), U_{ \pm}(k)$, and $T(k)$, respectively. Note that with this identification $t \in T^{J}(R)$ if and only if $t\left(\lambda_{\alpha}\right)=1$ for all $\alpha \in \Delta \backslash \Delta_{J}$. If $t \in T^{J}(R)$ and $C \subset \Phi_{J}^{+}$is a closed subset then $Y_{C}^{J}(t)=\left\{g \in G^{J}(R) ; g=x t y, x \in\right.$ $\left.U_{-}^{J}(k), y \in U_{C}(k)\right\}$ and $K_{C}^{J}(t)=U_{-}^{J}(R) \backslash Y_{C}^{J}(t) / U_{C}(R)$ are subsets of $Y_{C}(t)$ and $K_{C}(t)$, respectively.

From now on we assume that $R \subset k$ satisfies properties (P1) and (P2) (for example $\mathbf{Z} \subset \mathbf{Q}$, and $\mathbf{Z}_{p} \subset \mathbf{Q}_{p}$ satisfy (P1) and (P2)).

Proposition 2.8. Let $C$ be a closed subset of $\Phi^{+}$. (i) Let $C_{J}=C \cap \Phi_{J}$. If $t \in T^{J}(k)$ then $K_{C_{J}}^{J}(t)=K_{C}(t)$.

(ii) If $C \subset \Phi_{J}$ and $K_{C}(t)$ is not empty then $t\left(\lambda_{\alpha}\right)$ is a unit in $R$ for every $\alpha \in \Delta \backslash \Delta_{J}$.

Proof. Part (i) is an immediate consequence of Proposition 2.5. For part (ii) let $g=u^{\prime} t u \in Y_{C}(t)$ where $t \in T(k), u^{\prime} \in U_{-}(k)$, and $u \in U_{C}(k)$. Then $t\left(\lambda_{\alpha}\right) \in R, \alpha \in \Delta$, by Proposition 2.2, part (iii). Fix $\alpha \in \Delta \backslash \Delta_{J}$. We can write $u^{\prime}=x y$ where $x$ is a product of elements $U_{-\beta}, \beta \in \Phi^{+},\left\langle\lambda_{\alpha}, \beta\right\rangle=0$ and $y$ is a product of elements $U_{-\beta}, \beta \in \Phi^{+},\left\langle\lambda_{\alpha}, \beta\right\rangle>0$. Let $V$ be the irreducible representation of weight $\lambda_{\alpha}$ with a highest weight vector $v$. We can assume that $v$ belongs to a $\mathrm{Z}$-basis of an admissible lattice in $V$. Therefore $1 \otimes v$ belongs to a basis of $V(k)=k \otimes_{Z} M$. For any $g \in G(k)$, we let $f(g)$ denote the $1 \otimes v$-component of $g(1 \otimes v)$. Then

$$
f\left(g^{-1}\right)=f\left(u^{-1} t^{-1} y^{-1} x^{-1}\right)=f\left(u^{-1} t^{-1} y^{-1}\right)=t\left(-\lambda_{\alpha}\right)=\left(t\left(\lambda_{\alpha}\right)\right)^{-1} \in R
$$


since $x^{-1}(1 \otimes v)=1 \otimes v$ by Lemma 2.7 and since no sum of roots in $\Phi_{J}$ equals a sum of roots in $B(\alpha)$.

It can be shown that $P(w) \subseteq \Phi_{J}$ for any $w \in W_{J}$. Therefore we have

Corollary. If $w \in W_{J}$ and $t \in T^{J}(k)$ then $K_{w}^{J}(t)=K_{w}(t)$.

Definition. For any closed subset $C$ of $\Phi^{+}, t \in T(k)$, such that $K_{C}(t) \neq \varnothing$, and a pair of characters $\phi: U_{-}(k) \rightarrow\{z \in \mathbf{C},|z|=1\}$ and $\psi: U_{C}(k) \rightarrow\{z \in$ C, $|z|=1\}$ which are trivial on $U_{-}(R)$ and $U_{C}(R)$ respectively, we define

$$
S_{C}(t, \phi, \psi)=\sum \phi\left(u^{\prime}\right) \psi(u)
$$

where the summation ranges over a full set of representatives $u^{\prime} t u$ of $K_{C}(t)$, where $u^{\prime} \in U_{-}(k)$ and $u \in U_{C}(k)$ (note that $K_{C}(t)$ is finite, since we have assumed that $R \subset k$ satisfies property (P1)). We will call $S_{C}(t, \phi, \psi)$ a generalized Kloosterman sum associated with $C, t, \phi$ and $\psi$. We write $S_{w}(t, \phi, \psi)=$ $S_{P(w)}(t, \phi, \psi)$.

Example 2.9. Let $\Phi=\{\alpha,-\alpha\}$ be the root system of type $A_{1}, R=\mathbf{Z}$ and $k=\mathbf{Q}$. Then $G(\mathbf{Q})=S L(2, \mathbf{Q})$ and $T(\mathbf{Q}), U_{-}(\mathbf{Q}), U_{+}(\mathbf{Q})$ are, respectively, the diagonal, the lower triangular unipotent and the upper triangular unipotent subgroups of $S L(2, \mathbf{Q})$. A simple computation shows that if $w=\sigma_{\alpha}$,

$$
t=\left[\begin{array}{cc}
c & 0 \\
0 & \frac{1}{c}
\end{array}\right], \quad \phi\left(\left[\begin{array}{cc}
1 & 0 \\
y & 1
\end{array}\right]\right)=e^{2 \pi i m y} \quad \text { and } \quad \psi\left(\left[\begin{array}{cc}
1 & x \\
0 & 1
\end{array}\right]\right)=e^{2 \pi i n x}
$$

then $S_{w}(t, \phi, \psi)=S(-m, n, c)$ where $S(-m, n, c)$ is the classical Kloosterman sum.

Example 2.10. Let $R=\mathrm{Z}, k=\mathrm{Q}$ and let $\Phi$ be the root system of type $A_{r}$ in its standard representation as a subset of $\mathbf{R}^{r+1}$ (see [B or H, p. 64]). We identify the Weyl group $W$ of $\Phi$ with the group of permutation matrices in $G L(r+1, \mathbf{Q})$. Let $S\left(\theta_{1}, \theta_{2} ; t, w\right)$ be the $G L(r+1, \mathbf{Q})$ Kloosterman sum defined by formula (1.1). We define $w_{1}=w_{0} w^{-1} w_{0}^{-1}, t_{1}=w_{0} t w_{0}^{-1}, \phi(y)=$ $\theta_{1}\left(w_{0}^{-1} y w_{0}\right), y \in U_{-}(\mathbf{Q})$ and $\psi(x)=\theta_{2}\left(\left(w_{0} \omega\right)^{-1} y w_{0} \omega\right), x \in U_{w}(\mathbf{Q})$ where $w_{0}$ is the long element of the Weyl group (this is the unique element of $W$ sending each positive root to a negative root). Then $S_{w_{1}}\left(t_{1}, \phi, \psi\right)=S\left(\theta_{1}, \theta_{2} ; t, w\right)$ if the right-hand side of the equation is well defined. To verify this identity we may assume that the element $b_{2}$ is the definition of $S\left(\theta_{1}, \theta_{2} ; t, \omega\right)$ belongs to $U_{w}(\mathbf{Q})$. Consequently

$$
w_{0} b_{1} t \omega b_{2}\left(w_{0} \omega\right)^{-1}=w_{0} b_{1}\left(w_{0}\right)^{-1} w_{0} t\left(w_{0}\right)^{-1} w_{0} \omega b_{2}\left(w_{0} \omega\right)^{-1} \in Y_{w_{1}}\left(t_{1}\right)
$$

In fact the map $x \rightarrow w_{0} x\left(w_{0} \omega\right)^{-1}$ gives the desired bijection between $K_{w_{1}}\left(t_{1}\right)$ and $U_{+}(\mathbf{Z}) \backslash U_{+}(\mathbf{Q}) t \omega U_{+}(\mathbf{Q}) \cap S L(n+1, \mathbf{Z}) / U_{w}(\mathbf{Z})$.

Next proposition summarizes elementary properties of generalized Kloosterman sums.

Proposition 2.11. Let $C, t, \phi$ and $\psi$ be as in the definition of generalized Kloosterman sums.

(i) If $t \in T(R)$ then $S_{C}(t, \phi, \psi)=\#\left(K_{C}(t)\right)=1$.

(ii) If $t_{1} \in T(R)$ then

$$
S_{C}\left(t t_{1}, \phi, \psi\right)=S_{C}\left(t_{1}, \phi^{t}, \psi\right)=S_{C}\left(t_{1}, \phi, \psi^{t^{-1}}\right)
$$


where $\phi^{t}(x)=\phi\left(t x t^{-1}\right), x \in U_{+}(k), \psi^{t^{-1}}(y)=\psi\left(t^{-1} y t\right)$ and $y \in U_{C}(k)$.

(iii) Let $\vartheta$ be any automorphism or anti-automorphism of $G(k)$, defined over $R$, such that it preserves $T(k)$ and for any $\alpha \in \Phi^{+}$(resp. $\alpha \in \Phi^{-}$) and $\xi \in k$ one has $\vartheta\left(U_{\alpha}(\xi)\right)=U_{\vartheta(\alpha)}\left(r_{\vartheta} \xi\right)$ for some $r_{\vartheta} \in \mathbf{R}^{\times}$and $\vartheta(\alpha) \in \Phi^{+}$(resp. $\left.\vartheta(\alpha) \in \Phi^{-}\right)$. Then

$$
S_{C}(t, \phi, \psi)=S_{\vartheta(C)}\left(\vartheta(t), \phi_{\vartheta}, \Psi_{\vartheta}\right)
$$

where $\phi_{\vartheta}(x)=\phi\left(\vartheta^{-1}(x)\right), x \in U_{-}(k)$, and $\psi_{\vartheta}(y)=\psi\left(\vartheta^{-1}(y)\right), y \in U_{\vartheta(C)}(k)$.

The following proposition reveals when a Kloosterman sum related to a given root system $\Phi$ reduces to a Kloosterman sum related to a root subsystem $\Phi_{J}$. Notation is the same as in Proposition 2.8.

Proposition 2.12. Let $C, t, \phi, \psi$ be as in the definition of Kloosterman sums. We define $t_{1} \in T(k)$ by the formula $t_{1}\left(\lambda_{\alpha}\right)=t\left(\lambda_{\alpha}\right)$ if $\alpha \in \Delta_{J}$ and $t_{1}\left(\lambda_{\alpha}\right)=1$ for $\alpha \in \Delta \backslash \Delta_{J}$.

(i) If $C \subset \Phi_{J}$ then $t_{1} \in T(R)$ and $S_{C}(t, \phi, \psi)=S_{C}^{J}\left(t t_{1}^{-1}, \phi, \psi^{t_{1}^{-1}}\right)$ where the right-hand side is the Kloosterman sum in $G^{J}(k)$.

(ii) Let $t \in T^{J}(k)$ and let $C_{J}=C \cap \Phi_{J}$. Then $S_{C}(t, \phi, \psi) S_{C_{J}}^{J}(t, \phi, \psi)$.

The proposition follows directly from Propositions 2.8 and 2.11.

Corollary 2.13. Let $t, t_{1} \in T(R)$ be as in Proposition 2.12 and let $w$ be an element in $W$. Then $S_{w}(t, \phi, \psi)=S_{w_{J}}^{J}\left(t t_{1}^{-1}, \phi, \psi^{t_{1}^{-1}}\right)$ where $W_{J}$ is the unique element of $W_{J}$ such that $P(w) \cap \Phi_{J}$ (it can be shown that such element $w_{J}$ always exists).

This is a generalization of a result about $G L(r+1)$-Kloosterman sums obtained in [F, Proposition 3.6 and S, Corollary (3.11)].

\section{Global and local Kloosterman sums}

In this section we assume that either $R=\mathbf{Z} \subset k=\mathbf{Q}$ or $R=\mathbf{Z}_{p} \subset k=\mathbf{Q}_{p}$ where $p$ is a prime. We let $\Psi$ and $\Psi_{p}$ denote the fundamental characters of the additive group $k$, trivial on $R$, in the respective cases. Explicitly, if $k=\mathbf{Q}$ then $\Psi(x)=e^{2 \pi i x}$ and if $k=\mathbf{Q}_{p}$ then

$$
\Psi_{p}\left(\sum_{n=-N}^{\infty} a_{n} p^{n}\right)=e^{2 \pi i}\left(\sum_{n=-N}^{-1} a_{n} p^{n}\right) .
$$

We observe that if $x \in \mathbf{Q}$ then

$$
\Psi(x)=\prod_{p} \Psi_{p}(x) .
$$

Let $C$ be a closed subset in either $\Phi^{+}$or $\Phi^{-}$an let $U_{C}(k)$ be the corresponding subgroup of $G(k)$. We say that a group homomorphism $\varphi: U_{C}(k) \rightarrow\{z \in$ C; $|z|=1\}$ is a rational character, defined over $R$, if

$$
\varphi\left(\prod_{\alpha \in C} U_{\alpha}\left(\xi_{\alpha}\right)\right)=\Psi\left(\sum_{\alpha \in C} d_{\alpha} \xi_{\alpha}\right) \quad \text { where } d_{\alpha} \in R, \xi_{\alpha} \in k
$$

(the product is taken in a fixed order, and $\Psi$ is the fundamental character of $k / R)$. 
Remark. We say that a root in $C$ is indecomposable in $C$ if it is not a sum of roots in $C$. Let $D$ be the set of indecomposable roots in $C$. Then one can show that the commutator group $\left[U_{C}(k), U_{C}(k)\right]$ coincides with the group $U_{C \backslash D}(k)$. Consequently any rational character of $U_{C}(k)$ defined over $R$ is of the form

$$
\varphi\left(\prod_{\alpha \in D} U_{\alpha}\left(\xi_{\alpha}\right)\right)=\Psi\left(\sum_{\alpha \in D} d_{\alpha} \xi_{\alpha}\right) \quad \text { where } d_{\alpha} \in R, \quad \xi_{\alpha} \in k,
$$

and vice versa, given numbers $d_{\alpha} \in R, \alpha \in D$, then formula $(*)$ gives a rational character of $U_{C}(k)$. It is clear that if $C=\Phi^{+}$then $D=\Delta$. It has been pointed out to the author by V. Deodhar that in root systems whose elements are all of equal length, set $D$ for $C=P(w)$ is given by the formula $D=\left\{\alpha \in P(w), l\left(w \sigma_{\alpha}\right)=l(w)-1\right\}$, where $l(\cdot)$ is the length function on $W$. We observe that if $k=\mathbf{Q}$, then $\varphi(x)=\prod_{p} \varphi_{p}(x)$ where

$$
\varphi_{p}\left(\prod_{\alpha \in C} U_{\alpha}\left(\xi_{\alpha}\right)\right)=\Psi_{p}\left(\sum_{\alpha \in C} a_{\alpha} \xi_{\alpha}\right) .
$$

We will assume that the characters $\phi, \psi$ appearing in definition of generalized Kloosterman sums are rational, defined over $R$.

Proposition 3.1. Let $C$ be a closed subset of $\Phi^{+}$and let $t \in T(\mathbf{Q})$ and let $\phi, \psi$ be rational characters defined over $\mathbf{Z}$ of $U_{-}(\mathbf{Q})$ and $U_{C}(\mathbf{Q})$, respectively. Then

$$
S_{C}(t, \phi, \psi)=\prod_{p} S_{C}^{p}\left(t, \phi_{p}, \psi_{p}\right)
$$

$\left(S_{C}^{p}\right.$ denotes the $G\left(\mathbf{Q}_{p}\right)$ Kloosterman sum).

Proof. We first notice that if $p$ does not divide $t(\rho)$ then $t \in T\left(\mathbf{Z}_{p}\right)$ and $S_{C}^{p}\left(t, \phi_{p}, \psi_{p}\right)=1$ by Proposition 2.11. Therefore the proposition will follow if we show that the natural embedding

$$
K_{C}(t) \rightarrow \prod_{p \mid t(\rho)} K_{C}^{p}(t)
$$

is in fact a bijection. This fact is an immediate consequence of the following lemma.

Lemma 3.2. Let $C$ be a closed subset in either $\Phi^{+}$or $\Phi^{-}$and let $U_{C}\left(\mathbf{Q}_{p}\right)$ be the corresponding subgroup of $G\left(\mathbf{Q}_{p}\right)$. Let $S$ be a finite set of primes and let $x_{p} \in U_{C}\left(\mathbf{Q}_{p}\right)$ for $p \in S$. There exist elements $y, z \in U_{C}(\mathbf{Q})$ such that $x_{p} z$ and $y x_{p}$ belong to $U_{C}\left(\mathbf{Z}_{p}\right)$.

Proof of the lemma. Let $\left(\alpha_{1}, \alpha_{2}, \ldots, \alpha_{N}\right)$ be an ordering of $C$ such that $\left\{\alpha_{1}, \alpha_{2}, \ldots, \alpha_{q}\right\}$ is an ideal in $C$ for any $q, 1 \leq q \leq N$. We then have $x_{p}=U_{\alpha_{1}}\left(\xi_{1, p}\right) U_{\alpha_{2}}\left(\xi_{2, p}^{\prime}\right) \cdots U_{\alpha_{q}}\left(\xi_{q, p}\right)$ for some $\xi_{1, p}, \xi_{2, p}, \ldots, \xi_{q, p} \in \mathbf{Q}_{p}$. We proceed by induction on $q$. Clearly, the lemma holds if $q=1$. Assume $q>1$. We may suppose that $\xi_{q, p}=\xi \in \mathbf{Q}, p \in S$. Consequently, $U_{\alpha_{q}}(-\xi) x_{p}=$ $U_{\alpha_{1}}\left(\xi_{1, p}^{\prime}\right) U_{\alpha_{2}}\left(\xi_{2, p}^{\prime}\right) \cdots U_{\alpha_{q-1}}\left(\xi_{q-1, p}^{\prime}\right)$ for some $\xi_{1, p}^{\prime}, \xi_{2, p}^{\prime}, \ldots, \xi_{q-1, p}^{\prime} \in \mathbf{Q}_{p}$ by formula (2.1). By inductive hypothesis there exists $y^{\prime} \in U_{C}(\mathbf{Q})$ such that $y^{\prime} U_{\alpha_{q}}(-\xi) x_{p} \in U_{C}(\mathbf{Q})$ for all $p \in S$. Therefore $y=y^{\prime} U_{\alpha_{q}}(-\xi)$ satisfies the desired property. 
Corollary 3.3 (Multiplicativity of generalized Kloosterman sums). Let $t$ and $t^{\prime}$ be elements of $T(\mathbf{Q})$ such that $t(\rho)$ and $t^{\prime}(\rho)$ are relatively prime. Then

$$
S_{C}\left(t t^{\prime}, \phi, \psi\right)=S_{C}\left(t, \phi^{\prime}, \psi\right) S_{C}\left(t^{\prime}, \phi^{\prime \prime}, \psi\right)
$$

for some characters $\phi^{\prime}$ and $\phi^{\prime \prime}$.

Proof. By Propositions 3.1 and 2.11,

$$
S_{C}\left(t t^{\prime}, \phi, \psi\right)=\prod_{p \mid t(\rho)} S_{C}^{p}\left(t, \phi_{p}^{t^{\prime}}, \psi_{p}\right) \prod_{p \mid t^{\prime}(\rho)} S_{C}^{p}\left(t^{\prime}, \phi_{p}^{t}, \psi_{p}\right) .
$$

Since the character $\phi$ is rational, there exist $d_{\alpha} \in \mathbf{Z}, \alpha \in \Delta$, such that

$$
\phi(x)=\Psi\left(\sum_{\alpha \in \Delta} d_{\alpha} \eta_{\alpha}\right)
$$

for any $x=\prod_{\alpha \in \Phi^{+}} U_{-\alpha}\left(\eta_{\alpha}\right) \in U_{-}(\mathbf{Q})$. Consequently

$$
\phi^{t}(x)=\Psi\left(\sum_{\alpha \in \Delta} t(-\alpha) d_{\alpha} \eta_{\alpha}\right) \quad \text { and } \quad \phi^{t^{\prime}}(x)=\Psi\left(\sum_{\alpha \in \Delta} t^{\prime}(-\alpha) d_{\alpha} \eta_{\alpha}\right) .
$$

For each $\alpha \in \Delta$, we chose $b_{\alpha}, c_{\alpha} \in \mathbf{Z}$, such that

$$
b_{\alpha}-d_{\alpha} t(-\alpha) \in t^{\prime}\left(\lambda_{\alpha}\right) \mathbf{Z}_{p} \text { for all } p \mid t^{\prime}(\rho)
$$

and

$$
c_{\alpha}-d_{\alpha} t^{\prime}(-\alpha) \in t\left(\lambda_{\alpha}\right) \mathbf{Z}_{p} \text { for all } p \mid t(\rho) .
$$

Since $t(-\alpha)$ is in $\left.\mathbf{Z}_{p}\right)$ (resp. $t^{\prime}(\alpha)$ is in $\mathbf{Z}_{p}$ ) whenever $p \mid t^{\prime}(\rho)$ (resp. $p \mid t(\rho)$ ) such choice of $b_{\alpha}$ and $c_{\alpha}$ is possible by the Chinese Remainder Theorem. We define the characters $\phi^{\prime}$ and $\phi^{\prime \prime}$ by formulae $\phi^{\prime}(x)=\Psi\left(\sum_{\alpha \in \Delta} c_{\alpha} \eta_{\alpha}\right)$ and $\phi^{\prime \prime}(x)=\Psi\left(\sum_{\alpha \in \Delta} b_{\alpha} \eta_{\alpha}\right)$. Assume now that $x t y \in Y_{C}^{p}$ where $p \mid t(\rho)$. By Proposition $2.2 t\left(\lambda_{\alpha}\right) \eta_{\alpha} \in \mathbf{Z}_{p}$. Hence $\phi_{p}^{t^{\prime}}(x)=\phi^{\prime}(x)$ and

$$
S_{C}\left(t, \phi^{\prime}, \psi\right)=\prod_{p \mid t(\rho)} S_{C}^{p}\left(t, \phi_{p}^{t^{\prime}}, \psi_{p}\right) .
$$

By a similar reasoning we obtain

$$
S_{C}\left(t^{\prime}, \phi^{\prime \prime}, \psi\right)=\prod_{p \mid t^{\prime}(\rho)} S_{C}^{p}\left(t^{\prime}, \phi_{p}^{t}, \psi_{p}\right)
$$

and the proof is complete.

Corollary 3.6. Let $C$ be any closed subset of $\Phi^{+}$and let $t \in T(\mathbf{Q})$ satisfy $\left(t\left(\lambda_{\alpha}\right), t\left(\lambda_{\beta}\right)\right)=1$ for all $\alpha, \beta \in \Delta, \alpha \neq \beta$. Then

$$
S_{C}(t, \phi, \psi)=\prod_{\alpha \in \Delta} S\left(m_{\alpha}, n_{\alpha}, t\left(\lambda_{\alpha}\right)\right)
$$

where $n_{\alpha}=0$ if $\alpha \notin C, \psi\left(U_{\alpha}(\xi)\right)=\Psi\left(n_{\alpha} \xi\right), \xi \in \mathbf{Q}$, if $\alpha \in C$ and $m_{\alpha}=$ $-d_{\alpha} t\left(-\alpha+2 \lambda_{\alpha}\right), \alpha \in \Delta$. In particular (under the above assumptions about $t$ ) the Kloosterman sums $S_{w}(t, \phi, \psi), w \in W$ are products of classical Kloosterman sums.

Proof. Let $\alpha \in \Delta$ be fixed. We observe that $t\left(-\alpha+2 \lambda_{\alpha}\right)$ is an integer since $-\alpha+2 \lambda_{\alpha}=\sum_{\beta \in \Delta-\{\alpha\}}-\langle\alpha, \beta\rangle \lambda_{\beta}$ is a dominant weight. We define the element 
$t_{\alpha} \in T(\mathbf{Q})$ by the formula $t_{\alpha}\left(\lambda_{\beta}\right)=t\left(\lambda_{\beta}\right)^{\delta_{\alpha \beta}}, \alpha, \beta \in \Delta$. Proposition 3.3 implies that

$$
S_{C}(t, \phi, \psi)=S_{C}\left(t_{\alpha}, \phi^{\prime}, \psi\right) S_{C}\left(t\left(t_{\alpha}\right)^{-1}, \phi^{\prime \prime}, \psi\right)
$$

where $\phi^{\prime}(x)=\Psi\left(\sum_{\alpha \in \Delta} c_{\alpha} \eta_{\alpha}\right)$ and $\phi^{\prime \prime}(x)=\Psi\left(\sum_{\alpha \in \Delta} b_{\alpha} \eta_{\alpha}\right)$ with $b_{\alpha}$ and $c_{\alpha}$ determined by the formulae (3.4) and (3.5). In fact we can take $c_{\alpha}=d_{\alpha}\left(t t_{\alpha}^{-1}(-\alpha)\right)$ $=t\left(-\alpha+2 \lambda_{\alpha}\right), b_{\alpha}=0$ and $c_{\beta}=0, b_{\beta}=d_{\beta} t_{\alpha}(-\beta)$ for $\beta \in \Delta, \beta \neq \alpha$. Consequently, Example 2.9 and Proposition 2.8 imply

$$
S_{C}(t, \phi, \psi)=S\left(m_{\alpha}, n_{\alpha}, t\left(\lambda_{\alpha}\right)\right) S_{C^{\prime}}^{\prime}\left(t t_{\alpha}^{-1}, \phi^{\prime \prime}, \psi\right)
$$

where $S_{C^{\prime}}^{\prime}\left(t t_{\alpha}^{-1}, \phi^{\prime \prime}, \psi\right)$ is the Kloosterman sum for the group $G^{\prime}$ corresponding to the weight lattice of the root subsystem $\Phi^{\prime}$ of $\Phi$ whose base is $\Delta^{\prime}=$ $\Delta-\{\alpha\}$ and the closed set $C^{\prime}=C \cap \Phi^{\prime}$. We observe that if $\beta \neq \alpha$ then $t_{\alpha}(-\beta) t t_{\alpha}^{-1}\left(-\beta+2 \lambda_{\beta}\right)=t\left(-\beta+2 \lambda_{\beta}\right)$ and therefore the induction on the rank of $\Phi$ implies the statement of the corollary.

Example 3.7 (Kloosterman sums for the long element). We assume that $\Phi$ is an irreducible root system and we let $\alpha_{1}, \alpha_{2}, \ldots, \alpha_{r}$ be the basis of $\Phi$ as described in root systems tables in [B, Chapter VII]. We let $m_{i j}=\left\langle\alpha_{i}, \alpha_{j}\right\rangle$ be the Cartan integers. Suppose that $t, \phi, \psi$ are as in the definition of a Kloosterman sum, and we let $C=P\left(w_{0}\right)=\Phi^{+}$. We also put $d_{i}=d_{\alpha_{i}}$, $n_{i}=n_{\alpha_{i}}$, and $c_{i}=t\left(\lambda_{\alpha_{i}}\right), 1 \leq i \leq r$. If $c_{i}$ are pairwise relatively prime, Corollary 3.4 implies

$$
S_{w_{0}}(t, \phi, \psi)=\prod_{i=1}^{r} S\left(m_{i}, n_{i}, c_{i}\right) \quad \text { where } m_{i}=d_{i} \prod_{j \neq i} c_{j}^{-m_{i j}}, i=1,2, \ldots, r .
$$

For rank 2 root systems we have

$$
\begin{aligned}
& S_{w_{0}}(t, \phi, \psi)=S\left(-d_{1} c_{2}, n_{1}, c_{1}\right) S\left(-d_{2} c_{1}, n_{2}, c_{2}\right) \quad \text { if } \boldsymbol{\Phi} \text { is of type } \mathbf{A}_{2} \text {, } \\
& S_{w_{0}}(t, \phi, \psi)=S\left(-d_{1} c_{2}, n_{1}, c_{1}\right) S\left(-d_{2}\left(c_{1}\right)^{2}, n_{2}, c_{2}\right) \text { if } \Phi \text { is of type } \mathbf{B}_{2} \text {, } \\
& S_{w_{0}}(t, \phi, \psi)=S\left(-d_{1}\left(c_{2}\right)^{2}, n_{1}, c_{1}\right) S\left(-d_{2} c_{1}, n_{2}, c_{2}\right) \text { if } \boldsymbol{\Phi} \text { is of type } \mathbf{C}_{2} \text {, } \\
& S_{w_{0}}(t, \phi, \psi)=S\left(-d_{1}\left(c_{2}\right)^{3}, n_{1}, c_{1}\right) S\left(-d_{2} c_{1}, n_{2}, c_{2}\right) \text { if } \boldsymbol{\Phi} \text { is of type } \mathbf{G}_{2} \text {, } \\
& \text { if }\left(c_{1}, c_{2}\right)=1 \text {. }
\end{aligned}
$$

The problem of evaluation of Kloosterman sums in the general case (that is, if numbers $t\left(\lambda_{\alpha}\right), \alpha \in \Delta$, are not necessarily pairwise relatively prime) is much more difficult. The strongest known results concerning evaluation of $G L\left(n, \mathbf{Q}_{p}\right)$-Kloosterman sums can be found in [S, $\left.\S 4\right]$. Also in $[\mathrm{S}]$ a necessary and sufficient condition for existence of $G L\left(n, \mathbf{Q}_{p}\right)$-Kloosterman sums is given. Such a condition for Chevalley group Kloosterman sums is discussed in [D].

\section{REFERENCES}

[B] N. Bourbaki, Groupes et algebres de Lie, Chapitres 1, 4-6, 7-8, Hermann, Paris.

[B-F-G] D. Bump, S. Friedberg, and D. Goldfeld, Poincaré series and Kloosterman sums for Sl( $3, R)$, Acta Arith. 50 (1988), 31-89.

[Bo] A. Borel, Properties and linear representations of Chevalley groups, Seminar on Algebraic Groups and Related Finite Groups, Lecture Notes in Math., vol. 131, Springer-Verlag, Berlin-Heidelberg-New York, 1970. 
[Ch] C. Chevalley, Certain schemas de groupes semisimples, Sem. Bourbaki 1960-1961, Exposé 219, Benjamin, New York, 1966.

[D] R. Dabrowski, Integral points in a given Bruhat cell of a Chevalley group, preprint.

[D1] V. Deodhar, Some characterizations of Bruhat ordering on a Coxeter group and determination of the relative Möbius function, Invent. Math. 39 (1977), 187-198.

[D2] - Some characterizations of Coxeter groups, Enseign. Math. 32 (1986), 111-120.

[D-I] J.-M. Deshouiller and H. Iwaniec, Kloosterman sums and Fourier coefficients of cusp forms, Invent. Math. 70 (1982), 219-288.

[F] S. Friedberg, Poincaré series for GL(n): Fourier expansion, Kloosterman sums, and algebreogeometric estimates, Math. Z. 196 (1987), 165-188.

[H] J. E. Humphreys, Introduction to Lie algebras and representation theory, Springer-Verlag, 1980.

[K] B. Kostant, Groups over Z, Algebraic Groups and Discontinuous Groups, Proc. Sympos. Pure Math., vol. 9, Amer. Math. Soc., Providence, R.I., 1966.

[K1] H. Kloosterman, On the representation of numbers in the form ax $2+b y 2+c z 2+d t 2$, Acta Math. 49 (1926), 407-464.

[S] G. Stevens, Poincaré series on GL(r) and Kloosterman sums, Math. Ann. 277 (1986), 25-51.

[St] R. Steinberg, Lectures on Chevalley groups, mimeographed lecture notes, Yale Univ., New Haven, Conn., 1968.

Department of Mathematics, Indiana University, Bloomington, Indiana 47405 